\title{
Abordagens ao ensino das instrumentalidades na área de Ciência da Informação
}

\author{
Approaches to the education of the instrumentalities in the information science area
}

\author{
Ely Francina Tannuri de OliveiRA (1), João Batista Ernesto de MORAES (2), \\ Maura Duarte Moreira GUARIDO (3) y Mariângela Braga NORTE (4) \\ Faculdade de Filosofia e Ciencias, Unesp, Av. Higinio Muzzi Filho, 737 Marília, São Paulo, Brasil, CEP: \\ 17525-900, (1) etannuri@flash.tv.br (2) jota@marilia.unesp.br (3) mauraguarido@gmail.com (4) \\ mariangela@marilia.unesp.br
}

\begin{abstract}
Resumen
Esta investigación tuvo por objetivo evaluar la enseñanza de las asignaturas Lingüística y Documentación, Elementos de Lógica para Documentación, Métodos Cuantitativos Aplicados a la Ciencia de la Información e Inglés Instrumental, así como las relaciones que se establecen entre el contenido de éstas con la Ciencia de la Información. Para ello, se procedió a una reevaluación de los planes de enseñanza, así como a la articulación de estos con el Proyecto Pedagógico del Curso. Además, se encuestó a los alumnos con el objetivo de verificar la vinculación del contenido trabajado en las instrumentalidades con el área de Ciencia de la Información. Como resultados parciales, se obtuvo algunos puntos en común, articuladores de las instrumentalidades: a) integración de los contenidos con las demás asignaturas del curso; b) una práctica ajustada al tipo de profesional que se pretende formar; c) práctica de la averiguación y de la investigación; d) articulación de la enseñanza de graduación con la del postgrado. Como recomendación, se sugiere un examen de la distribución de las instrumentalidades en los currículos de los cursos del área de Ciencia de la Información de Iberoamérica para evaluar cómo esos currículos se comportan dentro de una perspectiva interdisciplinaria.
\end{abstract}

Palabras clave: Ciencia de la Información. Enseñanza. Materias instrumentales. Interdisciplinaridad.

\section{Introdução}

Como desdobramento do trabalho apresentado no Ibersid 2007 sobre as instrumentalidades no currículo do curso de Biblioteconomia, esta pesquisa teve por objetivo avaliar o ensino das disciplinas Métodos Quantitativos Aplicados à Ciência da Informação, Elementos de Lógica para Documentação, Lingüística e Documentação e Inglês Instrumental, todas sob responsabilidade dos autores, bem como as relações que se estabelecem entre o conteúdo destas com a Ciência da Informação.

\begin{abstract}
This research had the objective of evaluating the education of the disciplines Linguistics and Documentation, Elements of Logic for Documentation, Quantitative Methods Applied to the Information Science and Instrumental English, as well as the relations that they establish between its content and Information Science. In order to achieve such goal, a data collection instrument was applied to pupils with the objective of verifying the link between the content worked in the instrumentality and the Information Science area. As partial results, especially obtained by the analysis of four discipline summaries, as well as the analysis of the Political-Pedagogical Course Project, was got some points in common, joints of the instrumentality: a) contents integration with other course disciplines; b) a practice that goes with the clarity of the professional whom it intends to form; c) inquiry and research practice; d) joint of the graduation education with the pos-graduation. As a recommendation, is suggested a survey of the instrumentality distribution among the course curriculums of the Ibero-american Information Science area to evaluate how these curriculums behave inside an interdisciplinary perspective.
\end{abstract}

Keywords: Information Science. Education. Instrumentalities. Interdisciplinarity.

Os conteúdos das disciplinas em questão não objetivam a geração de conhecimentos nas áreas de origem, mas constituem-se, no entanto, instrumentos para a geração de conhecimento na área de Ciência da Informação. Além disso, há que se considerar a necessidade de uma articulação dos conteúdos com a prática profissional, rompendo-se com o modelo reprodutivo, no qual o docente apenas executa um programa já pronto, sem interações com a Ciência da Informação, propondo-se a construção de cursos que priorizem os conteúdos das instrumentalidades de forma mais pertinente à área de atuação do futuro profissional, de modo a articu- 
lar os conteúdos das diferentes disciplinas instrumentais com sua prática profissional, bem como a geração de conhecimento na área de Ciência da Informação.

Nesse sentido, a articulação dos conceitos das próprias disciplinas com os demais conceitos do curso em que estão inseridas, adquire importância, buscando-se uma maior contextualização e interfaces, uma vez que os conteúdos não devem valer como um fim em si mesmos, mas, na medida em que se integram internamente, convergir para objetivos mais amplos, vinculados com a prática social global do profissional da informação. Considera-se ainda que, os educadores de um mesmo curso tenham uma prática condizente com a clareza do profissional que se pretende formar, não se restringindo apenas à competência técnica. Neste sentido, a interdisciplinaridade torna-se uma importante aliada na reconstituição da unidade dos conhecimentos dispersos, integrando-os com as diversas realidades.

Assim, a formação dos profissionais da área de Ciência da Informação, não pode prescindir de outras áreas, como também acontece nos diferentes campos científicos, considerando-se a não compartimentalização do saber, bem como a própria epistemologia da área.

Justifica-se essa pesquisa, especialmente pela carência de estudos no ensino das disciplinas quando tratadas como instrumentalidades para outras áreas do conhecimento.

\section{Procedimentos metodológicos}

Como procedimento metodológico, em um primeiro momento realizou-se a reavaliação dos planos de ensino e sua articulação com o Projeto Político Pedagógico do Curso, que sempre houve a interdisciplinaridade como meta, sem perder, no entanto, o caráter disciplinar. Além disso, foi aplicado um instrumento de coleta de dados junto aos alunos de graduação em Arquivologia e Biblioteconomia, com o objetivo de verificar a vinculação do conteúdo trabalhado nas instrumentalidades com a área de Ciência da Informação. Como resultados obtidos, especialmente da análise das ementas das quatro disciplinas, bem como do Projeto Político Pedagógico do Curso e das respostas obtidas nos questionários, alguns pontos em comum, articuladores das instrumentalidades, foram obtidos:

a) integração dos conteúdos com as demais disciplinas do curso, visando a interagir com os objetivos maiores da área de Ciência da Informação; b) uma prática condizente com a clareza do profissional que se pretende formar, não se restringindo apenas à competência técnica, mas tendo-se em vista a formação integral do profissional da informação;

c) prática da investigação e da pesquisa, considerando-se que esta é um dos objetivos maiores do curso, pois o ensino não pode sobreviver sem a pesquisa sob pena de tornar-se elemento de mera reprodução conteudística;

d) articulação do ensino de graduação com o da pós-graduação, seja pela perspectiva de continuidade de uma trajetória de pesquisa iniciada na graduação pelos discentes, seja pela possibilidade da pós-graduação oferecer maior contato com o estado-da-arte em Ciência da Informação, integrando atividades de pesquisa, cursos e palestras com profissionais da instituição, bem como de outras instituições nacionais e internacionais.

A partir destes pontos articuladores das quatro instrumentalidades, analisaram-se as diferentes ênfases dadas a cada uma das disciplinas.

\section{Abordagem no ensino das diferentes instrumentalidades}

\subsection{Metodologias Quantitativas Aplicadas à Ciência da Informação}

A disciplina Metodologias Quantitativas Aplicadas à Ciência da Informação, especialmente quando tratadas como ferramentas para o desenvolvimento das outras áreas do conhecimento, merecem uma particular atenção por parte dos pesquisadores e educadores, considerando que, em geral, elas constituem-se o único ferramental para pesquisa quantitativa que os alunos terão em sua formação, em nível de graduação. A disciplina em questão compreende os seguintes conteúdos: Estatística Aplicada à $\mathrm{Ci}$ ência da Informação, Bibliometria, Tratamentos métricos para avaliação da produção científica e demais indicadores numéricos que se prestam a análises quantitativas na Ciência da Informação.

Para tal, considera-se que a disciplina deve tratar de questões da realidade do campo de atuação dos alunos, de forma a instigá-los na percepção de como as quantificações estão inseridas nos diversos fazeres do profissional da informação.

Desse modo, especialmente quando trabalhada como disciplina de natureza instrumental, o ato de vincular o conhecimento numérico ao universo de conhecimento do aluno requer do docente a compreensão do campo para o qual se propõe a ser instrumento, integrando os conceitos da 
própria disciplina com os demais conceitos do curso em que está inserida.

Sowey (1995) destaca que ensinar coerentemente Estatística Aplicada significa inseri-la em um todo maior. A partir do momento em que os alunos conhecem e compreendem os tratamentos estatísticos, percebendo suas implicações e significações no todo em que se insere, alargase a possibilidade de os conhecimentos comporem a estrutura cognitiva e serem duradouros.

\section{Potter (1995, p. 260) destaca que}

o objetivo mais importante de um curso de Estatística é encorajar os estudantes a serem praticantes deste instrumental. O conhecimento estatístico definitivamente nada significa se ele não se relaciona a questões e problemas reais.

A partir da relevância de se trabalhar com dados reais, advindos da própria pesquisa, proposta esta desenvolvida em trabalho já publicado anteriormente (Grácio e Oliveira, 2004), recorreuse à prática da investigação e da pesquisa quantitativa como procedimento de trabalho. Os alunos desenvolvem mini-projetos de pesquisa por meio da coleta dados concernentes a questões do interesse do seu cotidiano profissional, buscando a associação dos conteúdos estatísticos com a área de atuação do aluno. Com base nestas questões, os estudantes constituem pequenos grupos e elaboram um projeto de pesquisa. Cada grupo de estudantes procede então, a construção do instrumento para a coleta e organização dos dados, elaboração de tabelas e gráficos, cálculo dos principais parâmetros estatísticos adequados, bem como a análise e interpretação dos resultados.

Esta prática encontra respaldo nos trabalhos de Gnanadesikan et al. (1997) e Garfield (1993) que permitem uma reflexão sobre questões da prática do ensino de Metodologias Quantitativas nos cursos de graduação. Os autores enfocam o uso de aprendizagem ativa e cooperativa como essencial. Neste processo, os estudantes, em grupos, trabalham com atividades concretas baseadas em problemas do mundo real, construindo seus próprios conhecimentos.

Ainda em relação à questão da articulação do ensino da graduação e da pós-graduação, nos conteúdos relativos à Bibliometria e tratamentos métricos em geral, propõe-se um tema de pesquisa, com trabalhos em iniciação científica na graduação, tomando maior consistência na pósgraduação, na qual é oferecida uma disciplina especialmente relativa a estudos métricos.

\subsection{Elementos de Lógica para Documentação}

Em relação à disciplina Elementos de Lógica para Documentação, destaca-se a pertinência da Lógica, como ciência que induz regras e ensina o uso das mesmas para o homem atingir a verdade, enquanto uso da ordem nas operações da razão. É uma ciência das leis ideais do pensamento, é a arte de aplicá-las corretamente à procura e à demonstração da verdade. É, portanto, a ciência da ordem a ser estabelecida nas operações da razão.

A Lógica ensinada para os alunos do $1^{\circ}$ ano de Biblioteconomia é de uma aplicação prática, pois os objetivos na disciplina constituem-se em utilizar procedimentos de análise e síntese tendo como base parâmetros da Lógica Formal, desenvolver o raciocínio e manter coerência na sua exposição, bem como distinguir formas de representar informação e conhecimento.

Partindo das regras estabelecidas pela Lógica Formal é que se inicia a disciplina destacando os conceitos de Idéia e Termo, Juízo e Proposição, o Raciocínio e a Argumentação.

A cada divisão da Lógica Formal, apresentamse artigos e textos para que os alunos construam sua habilidades para identificar a Idéia ou conceitos utilizados pelo autor, para que, posteriormente, utilize-os na Análise Documentária no momento da representação dos assuntos.

A partir da identificação do conceito, passa-se à noção de Juízo, que é a operação pela qual a inteligência une o predicado ao sujeito pela afirmação ou separa pela negação. Neste momento, os alunos deverão perceber a idéia do autor do texto e emitir juízo para que, em seguida, através da expressão verbal, apresentem a proposição dada pelo autor, demonstrando que, de um juízo conhecido, passa-se para outro juízo desconhecido, estimulando o raciocínio para concluir com a compreensão textual.

Destaque-se, com relevância, que todos os textos e artigos, utilizados na disciplina, foram selecionados para o uso comum nas disciplinas Expressão Escrita em Língua Portuguesa e Análise Documentária e Elementos de Lógica para Documentação, consolidando, desta forma, as questões articuladoras entre os conteúdos que serviram como premissa para este estudo.

Registre-se ainda que a aplicação prática da disciplina não se restringe somente a textos, mas também a filmes, peças teatrais, letras de músicas, etc., na busca de compreensão e desenvolvimento do raciocínio coerente em sua exposição oral e escrita na distinção de formas 
de representação do conhecimento e da informação.

\subsection{Lingüística e Documentação}

A Análise Documental tem por objetivos estabelecer uma ponte entre o usuário e o documento, fornecer subsídios ao processo de disseminação da informação, e gerar produtos documentários (resumos e índices). (Guimarães, 2003, p. 104). Para tanto, e dada sua natureza disciplinar, a Análise Documental, como destaca o referido autor (p. 109), necessita recorrer ao aparato teórico de outras áreas, como a Lingüística, para que possa explicitar os próprios procedimentos.

A Lingüística, definida por Orlandi (2003, p. 9) como o estudo científico que visa descrever ou explicar a linguagem verbal humana, oferece uma grande contribuição para a Análise Documental, principalmente pelo fato de a representação do conteúdo ocorrer por meio de metalinguagens denominadas Linguagens Documentais, que congregam elementos de natureza sintática e semântica (Guimarães, 2003, p. 109).

Neste sentido, pode-se perceber que a Análise Documental recorre à Lingüística em busca de elementos e conceitos possíveis de serem utilizados no seu fazer pragmático conforme afirma Cunha (1989, p. 50).

Desta forma, o objetivo da disciplina Lingüística e Documentação é o de fornecer aos alunos as bases de constituição da ciência lingüística, sempre com a preocupação de destacar as interfaces entre a Lingüística e a Documentação.

Para tanto, parte-se da definição de língua e de linguagem, buscando-se com esta diferenciação destacar o objeto de estudos da Lingüística.

Na seqüência do curso, são focadas as dicotomias saussurianas (Significante e Significado, Sintagma e Paradigma, Língua e Fala) com o objetivo de melhor compreender alguns aspectos da Análise Documental.

Um destes aspectos estudados é a questão da conversão da linguagem natural em linguagem documental. Para Gardin (1969) é essencial a linguagem documental para converter o conteúdo dos textos em informação e, para tanto, foi proposto um modelo de linguagem, constituída por um conjunto de termos (o léxico), por relações entre as unidades lexicais, determinadas a priori (o eixo paradigmático) e por uma sintaxe que articula os encadeamentos entre os termos da linguagem, face a um documento específico (o eixo sintagmático) (Gardin, 1973).
Nota-se claramente nesta definição a influência de uma das dicotomias saussurianas (Sintagma $X$ Paradigma), o que evidencia, também, que a base das teorias gardinianas sobre $A D$ repousa em teorias lingüísticas, o que reforça o caráter instrumental da disciplina Lingüística e Documentação.

$\mathrm{Na}$ seqüência do curso, é estudado o signo lingüístico, focando-se as visões de Saussure, Emile Benveniste e Hjelmslev.

Buscando-se um maior aprofundamento nos estudos lingüísticos, são vistas as várias abordagens lingüísticas tais como a Morfologia, a Sintaxe, a Semântica, e o Léxico.

$O$ encerramento do curso se dá com uma visão das definições Terminológicas, bem como são abordadas algumas noções de Lexicologia, sempre com a preocupação de evidenciar o enlace entre a Lingüística e a Documentação.

\subsection{Inglês Instrumental: Leitura}

Vale ressaltar mais uma vez, que na área da Ciência da Informação a necessidade de interface com outras áreas de conhecimento, reflete significativamente na formação dos alunos e o diálogo multidisciplinar traz benefícios que consolidam a prática profissional.

No atual mundo globalizado, com inúmeras formas de intercâmbios internacionais, faz-se indispensável a aprendizagem de línguas estrangeiras, pois é um instrumento necessário de comunicação e conhecimento.

Sendo assim, a disciplina Inglês Instrumental: Leitura é sem dúvida, imprescindível para busca de informações, ampla comunicação entre indivíduos e troca de conhecimentos entre diversas sociedades.

Para os estudantes e profissionais da Ciência da Informação que trabalham acessando bases de dados, registrando e organizando informações com a finalidade de disponibilizá-las para seus usuários, a leitura em língua inglesa tornase imprescindível, pois uma grande maioria dos textos técnicos e científicos está escrito nesse idioma.

A disciplina Inglês Instrumental: Leitura para os alunos da Ciência da Informação visa auxiliá-los nas tarefas de acesso, processamento e tratamento das informações científicas. É uma ferramenta de trabalho no seu dia a dia.

Nesse curso, a partir de textos autênticos, técnicos, científicos e de assuntos gerais, os aprendizes são expostos a estratégias de leitura, ao ensino de aspectos morfológicos, sintáticos e 
lexicais da língua com a finalidade de capacitálos a uma compreensão em vários níveis: compreensão geral, compreensão de pontos principais, leitura detalhada, leitura crítica e elaboração de resumos.

A metodologia utilizada no curso visa também a fornecer condições para que os alunos possam experimentar diferentes contextos de aprendizagem, favorecendo-os nos diferentes estilos e estratégias de aprendizagem. Para isso, optouse pela utilização do Blended Learning que integra o ensino presencial e on line - a distância, como trabalho extra-classe, permitindo, assim, oferecer ao aluno maior integração com as tecnologias de informação e comunicação, tão necessárias na atualidade.

Com esses avanços, as informações são registradas em suportes físicos e digitais (multimídia/ internet/ hipertexto) que modificaram as formas de leitura, tornando-as mais complexas e, nesse sentido, também devem ser ensinadas as novas possibilidades de compreensão desses novos formatos.

É inegável a importância da leitura em língua inglesa dentro de um processo de ensino e aprendizagem de comunicação e informação, pois abre possibilidades para a pesquisa, proporciona crescimento intelectual e profissional.

\section{Considerações finais}

Algumas considerações merecem destaque. Pôde-se perceber que todas as disciplinas instrumentais apresentadas articulam seus conteúdos com a Ciência da Informação, investigam e pesquisam novos procedimentos, bem como apresentam grande preocupação na elaboração de um respaldo teórico para o ensino das disciplinas em questão. Assim, devem ser citados os trabalhos publicados, pelos autores, sobre 0 ensino de Metodologias Quantitativas, em revistas da área, como a revista SBEM (Sociedade Brasileira de Educação Matemática) e revista DataGramaZero, específica na área de Ciência da Informação, o ensino por projetos, bem como os textos e artigos, utilizados nas disciplinas, selecionados para o uso comum nas disciplinas Expressão Escrita em Língua Portuguesa e Análise Documentária e Elementos de Lógica para Documentação, consolidando as questões articuladores, que serviram como premissa para esses estudos.

Ainda em relação especialmente à disciplina Lingüística e Documentação busca-se diferenciar a linguagem natural de linguagem documental, destacando-se as interfaces entre a Lingüística e a Documentação, além da Análise Docu- mental, dada sua natureza disciplinar, recorrer ao aparato teórico de outras áreas para explicitar os próprios procedimentos, confirmando mais uma vez as questões articuladoras entre as instrumentalidades e Ciência da Informação.

Em inglês instrumental, optou-se pela utilização do Blended Learning que integra o ensino presencial e on line - a distância, como trabalho extra-classe, permitindo assim, oferecer ao aluno maior integração com as tecnologias de informação e comunicação.

Ainda em relação às considerações finais, devese ressaltar que as análises e as avaliações relativas a cada disciplina em questão encontram-se em andamento, bem como a aplicação dos instrumentos de avaliação junto aos discentes. Os resultados até aqui obtidos apontam que, a formação dos profissionais da área de Ciência da Informação não pode prescindir de outras áreas, considerando-se a não compartimentalização do conhecimento, bem como a própria epistemologia desse campo de estudos. Como recomendação, sugere-se um levantamento da distribuição das instrumentalidades nos currículos dos cursos da área de Ciência da Informação da Ibero-América para avaliar como esses currículos se comportam dentro de uma perspectiva interdisciplinar.

\section{Referências}

Cappelletti, I.F. (1992). A docência no ensino de $3^{\circ}$ grau. // D'Antola, A. A prática docente na universidade. São Paulo: EPU, 1992. 3-12.

Cunha, I.M.R.F. (1989). Análise documentária. // Smit, J. W. (Coord.). Análise documentária: a análise da síntese. Brasília: IBICT, 1989.

Demo, P. (1993). Desafios modernos da Educação. Petrópolis: Vozes, 1993.

Gannadesikan, M.; Scheaffer, R.L; Watkins, A. E.; Witmer,J.A. (1997). An activity-based Statistics course. // Journal of Statistics Education. 5:2 (1997). http://www.amstat.org/publications/jse/ (09 de abril de 2008).

Gardin, J.-C. (1969). Semantic analysis procedures in the sciences of man. (1989). // Social Science Information. 8:1 (1969) 17-42.

Garfield, J. (1993). Teaching Statistics using small - group cooperative learning. // Journal of Statistics Education. 1:1 (1993). http://www.amstat.org/publications/jse/ em: 09 de abril de 2008.

Guimarães, J. A. C. (2003). A análise documentária no âmbito do tratamento da informação: elementos históricos e conceituais. // Rodrigues, G. M.; Lopes, I. L. (Org.). Organização e representação do conhecimento na perspectiva da Ciência da Informação. Brasília: Thesaurus, 2003. 100-118.

Grácio, M.C.C; Oliveira, E.F.T. (2004). O ensino de Estatística na UNESP/ Campus de Marília. // Educação Matemática em revista - SBEM. 17 (2004) 9-15.

Lück, H. (1995). Pedagogia interdisciplinar: fundamentos teórico-metodológicos. Petrópolis: Vozes, 1995. 
Oliveira, E. F. T.; Moraes, J.B.E.; Guarido, M.D.M. (2007). Estudio evolutivo de las asignaturas del Núcleo de Formación General y de Instrumentalidades del Currículo Pleno del Curso de Biblioteconomia de la Facultad de Filosofia y Ciencias de la UNESP - Marília. // García Marco, F.J.(Org.). Avances y perspectivas en sistemas de información y documentación en el entorno digital. Zaragoza: Prensas Universitarias de Zaragoza, 2007. 239-244.

Oliveira, E.F.T. et al. (2008). Planos de ensino das disciplinas Lingüística e Documentação, Elementos de Lógica para Documentação, Métodos Quantitativos Aplicados à Ciência da Informação e Inglês Instrumental. Universidade Estadual Paulista "Júlio de Mesquita Filho". Faculdade de Filosofia e Ciências. Departamento de Ciência da Informação, Marília, 2008.

Orlandi, E.P. (2003). O que é lingüística. São Paulo: Brasiliense, 2003.

Potter, A.M. (1995). Statistics for Sociologists: Teaching Techniques that Work. // Teaching Sociology. 23 (1995) 259-263.

Projeto político pedagógico do curso de Biblioteconomia. (2003). Universidade Estadual Paulista "Júlio de Mesquita Filho". Faculdade de Filosofia e Ciências. Departamento de Ciência da Informação, Marília, 2003.

Sowey, E. R. (1995). Teaching Statistics: making it memorable. // Journal of Statistics Education. 3:2 (1995). http://www.amstat.org/publications/jse/ (09 de abril de 2008).

Oliveira, Ely Francina Tannuri de; Moraes, João Batista Ernesto de; Guarido, Maura Duarte Moreira; Norte, Mariângela Braga. Abordagens ao ensino das instrumentalidades na área de Ciência da Informação. // Ibersid. (2008) 325-330. ISSN 1888-0967. 